\title{
Vinculación laboral en personas de la tercera edad: el caso de los empacadores voluntarios en tiendas de autoservicio
}

\section{Employment bonding in the elderly: the case of volunteer's packers in supermarkets}

\author{
Alberto Pérez-Fernández y José Apolonio Venegas-Venegas \\ Facultad de Ciencias Económicas-Administrativas de la Universidad \\ Autónoma del Carmen, México \\ Facultad de Ciencias Agronómicas de la Universidad Autónoma de Chiapas, \\ México
}

\section{Resumen}

Este artículo tiene por objetivo estudiar las características de 50 adultos mayores que laboran como empacadores voluntarios en cuatro centros comerciales en Ciudad de Carmen, Campeche, México. Para el análisis de las variables estudiadas se emplearon tablas de contingencia y regresión lineal múltiple. Los resultados muestran que en promedio una persona obtiene 31 pesos por cada hora que labora y aumenta 1.06 pesos por cada cliente atendido. La principal razón por la que las personas adultas continúa trabajando es porque no recibe ingresos suficientes (ya sea pensión o apoyo gubernamental). Las políticas sociales cubren con deficiencia este sector de la población y obliga a las personas mayores a mantenerse en el mercado laboral.

Palabras clave: Adulto mayor, ingresos, regresión lineal, calidad de vida.

Abstract

The objective of this article was to study characteristics of 50 senior citizen working as volunteer packers in four supermarkets in Ciudad del Carmen, Campeche, Mexico. For the analysis of the variables, contingency tables and multiple linear regression were used. Results shown that on average, a person earns 31 pesos for each hour worked and increases 1.06 pesos for each customer served. The main reason that older persons continue to work is the lack of income (either pension or government support). Social policies poorly cover this sector and this situation forces many older people to work.

Keywords: Older people, income, lineal regression, quality of life. 


\section{INTRODUCCIÓN}

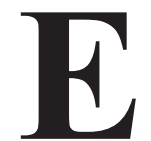

1 objetivo del presente artículo fue analizar las características de 50 personas de la tercera edad que se encuentran laborando como empacador voluntario en cuatro tiendas de autoservicio en Ciudad del Carmen, Campeche. Los aspectos estudiados fueron referentes a la edad, nivel educativo, dependientes económicos, razones de inserción en las actividades productivas, enfermedades y problemas de salud. Las personas entrevistadas forman parte de la base de datos del Instituto Nacional de las Personas Adultas Mayores (INAPAM) en Ciudad del Carmen, Campeche, el cual se encarga de identificar y dirigir a las personas a las tiendas y negocios que requieren ayudantes como empacadores voluntarios.

El estudio de este sector poblacional cobra importancia debido a que el proceso de crecimiento y envejecimiento de la población de México es evidente. Para el año 2018 el índice de envejecimiento fue de 27.34 por ciento y para 2030 se pronostica un índice de 45.87 por ciento Consejo Nacional de Población (CONAPO, 2019). El aumento en el número de ancianos con respecto al de personas en edad de trabajar implicará una significativa carga económica para la población económicamente activa ocupada (Esquivel y Sánchez, 1995); además, el incremento en la longevidad involucra contextos complejos para los sistemas de protección social y salud económica de la nación (Filgueira y Espíndola, 2015:25). Cuando el país no opera programas de protección social, mantener a los adultos mayores en el sistema productivo significa un menor peso para la carga fiscal del país (Oelckers, 2015).

Existen estudios realizados que confirman el envejecimiento paulatino de la población y la necesidad de conocer las fortalezas de la población mayor y sus capacidades para mantenerse productivos (Oelckers, 2015); y es que de acuerdo con los modelos jurídicos reclamantes de la protección a las personas longevas en acatamiento de la cláusula Pacta SuntServanda y el principio Pro-Homine, los adultos mayores deben ser protegidos para tener una vida digna (Romano, 2013: 2). Cuando las personas no tienen las capacidades para mantenerse por sí mismos, el Estado debe intervenir para proveer las condiciones de trabajo o de programas sociales que generen ingresos y satisfacción a la población.

El mercado de trabajo en México exige personas jóvenes y con alta productividad; sin embargo, el aumento de adultos mayores que demandan ingresos y servicios para satisfacer sus necesidades básicas, exigen cambios estructurales, sociales, legales y políticos que modifiquen al mercado 
laboral. Existe una situación vulnerable de la población de más de 60 años debido a las circunstancias culturales y económicas que en ocasiones reflejan pobreza, abandono y marginalidad por parte de la familia o de la sociedad (Navarro, 2017: 5). La seguridad social en México protege de manera efectiva a una proporción muy baja de personas longevas, y es que la insuficiencia de una pensión orilla a este sector a continuar trabajando (Damián, 2016), generalmente permanecen dinámicos en actividades del hogar, independientes mediante pequeños negocios, trabajos agrícolas o trabajos informales (Yánez, Maldonado y Del Risco, 2016).

En el caso de México, hay deficiencias institucionales y culturales para integrar socialmente a la población de la tercera edad (Bruno y Acevedo, 2016); la poca aceptación en ocasiones se debe a que existen algunas capacidades cognitivas que disminuyen con los años; sin embargo, otros aspectos como el pensamiento pragmático, pensamiento integrador y práctico mejoran conforme la persona envejece (Maldonado, Corrales, Ruíz y González, 2016). Como país en desarrollo y con programas asistencialistas en proceso de consolidación no asegura un sistema de calidad de vida y un ingreso conforme a las necesidades de la sociedad mexicana, así pues, los grupos vulnerables como los adultos mayores deben considerar un periodo más largo de permanencia en las actividades económicas. Los factores que influyen en la permanencia de los ancianos en el mercado laboral son el sexo, las pensiones, el área del hogar del anciano, el estado de salud y la jefatura de hogar (Yánez et al., 2016: 61).

\section{Calidad de vida en adultos mayores}

La calidad de vida es un atributo de la persona, se percibe como el grado de una sociedad para posibilitar la satisfacción de las necesidades materiales y no materiales de las personas; es una respuesta situacional o un estado sentimental con un enfoque sociológico y económico (Durán, Prieto y García, 2017; Villasmil, Romero, Chivetta y Palacios, 2017). La calidad de vida definida en el Foro Mundial de la Salud de 1996 considera seis esferas: físico, psicológico, grado de dependencia, relaciones sociales, entorno y aspecto espiritual (OMS, 1996). El cumplimiento de estos elementos refleja la opinión propia de las personas de acuerdo a su percepción; sin embargo, no se tiene calidad de vida si las personas consideran que una de las facetas pertenecientes a cada esfera no se cumple (Tabla 1). 
Tabla 1: Esferas de la calidad de vida de una persona

\begin{tabular}{|c|c|}
\hline Esferas & Facetas \\
\hline Físico & $\begin{array}{l}\text { Dolor y malestar } \\
\text { Energía y fatiga } \\
\text { Sueño y Descanso }\end{array}$ \\
\hline Psicológico & $\begin{array}{l}\text { Sentimientos positivos } \\
\text { Labor de reflexión } \\
\text { Memoria, Concentración, Aprendizaje } \\
\text { Autoestima } \\
\text { Imagen y apariencia corporal } \\
\text { Sentimientos negativos }\end{array}$ \\
\hline Grado de independencia & $\begin{array}{l}\text { Movilidad } \\
\text { Actividades de la vida diaria } \\
\text { Dependencia a medicaciones } \\
\text { Capacidad de trabajo }\end{array}$ \\
\hline Relaciones sociales & $\begin{array}{l}\text { Relaciones personales } \\
\text { Apoyo social } \\
\text { Actividad sexual }\end{array}$ \\
\hline Entorno & $\begin{array}{l}\text { Seguridad física } \\
\text { Entorno doméstico } \\
\text { Recursos financieros } \\
\text { Atención sanitaria } \\
\text { Oportunidades para adquirir información } \\
\text { Actividades recreativas } \\
\text { Entorno físico (contaminación, ruido, tráfico) } \\
\text { Transporte }\end{array}$ \\
\hline Espiritual & $\begin{array}{l}\text { Religión } \\
\text { Creencias personales }\end{array}$ \\
\hline
\end{tabular}

Fuente: OMS, 1996.

El concepto de calidad de vida se utiliza en el contexto médico; sin embargo, también considera un enfoque social y económico que le permite a las personas satisfacer sus necesidades de tipo material (Rubio, Rivera, Borges y González 2015: 4). Los individuos al tener seguridad económica pueden adquirir alimento, vestido, vivienda, medicinas y elementos recreativos que implican mejoras en todos los aspectos de la vida de los adultos mayores.

La esfera de grado de independencia considera la capacidad de trabajo como una variable importante, y es que, de acuerdo con diversos estudios, las personas mayores se enfrentan a jornadas laborales inadecuadas, con poca cobertura de seguridad social y salarios bajos, obligándolos a reconocer su vulnerabilidad y dependencia económica de familiares; compro- 
metiéndolos a realizar actividades en casa o tienen que seguir trabajando como único recurso para subsistir (Rendón 2004; De los Santos y Carmona; 2018). El trabajo forma parte de la vida diaria de los adultos mayores, al ser una actividad física se traduce en una mejora del estado anímico, evita el aislamiento y mejora las relaciones sociales (Limón y Ortega, 2011: 232). La calidad de vida involucra el desarrollo de actividades en todas las áreas que permitan a la persona sentirse plena con lo que hace de acuerdo con sus capacidades físicas, económicas y psicológicas.

\section{AdULTOS MAYORES Y VINCULACIÓN PRODUCTIVA}

Un adulto mayor se considera como toda persona que alcanza los 60 años de edad y los supera (Alonso et al., 2007), en general, se caracteriza por la aparición de varios estados de salud complejos denominados síndromes geriátricos y son consecuencias de factores subyacentes que incluyen fragilidad, estados delirantes, dolores de espalda y cuello, neumopatías obstructivas crónicas, diabetes y demencia (OMS, 2018). Estos elementos asociados a la edad no implican que la persona deje de hacer actividades en el transcurso de su vida, por el contrario, el uso de la experiencia, conocimientos y sensibilidad de un adulto permite desarrollar más y mejores actividades permitiendo a la persona una plenitud por sentirse útil para la empresa, familia o sociedad. Cuando se les niega la posibilidad de participación de interrelación y de decisión por el hecho de sus limitaciones físicas y mentales, se contribuye a un envejecimiento visto como algo negativo (Alessandrini y Sánchez, 2007: 6).

El contexto socioeconómico de la región latinoamericana representado por desigualdades sociales, alta incidencia de pobreza, poca cobertura de asistencia social y una tendencia global al deterioro de las redes de apoyo familiar, es factible que una alta proporción de adultos mayores continúen participando en la fuerza laboral (Arcia, 2004: 28). La población que deja de trabajar genera una inadaptación a la jubilación que puede inducir depresión en las personas (Llanes, López, Vázquez y Hernández, 2015: 65). Una opción para apoyar económica y psicológicamente a los adultos mayores es por medio de la inclusión en actividades físicas, sociales y productivas.

El trabajo se puede definir como una actividad física y una forma de interacción grupal, que además proporciona una retribución económica acorde al desgaste físico o mental que sufre la persona y la contribución que hace al proceso productivo. Para Levaggi (2004) y haciendo uso del Tesauro de la Organización Internacional del Trabajo (OIT) define el tra- 
bajo como un conjunto de actividades que realizan las personas ya sea que reciban una remuneración o no, pero que tienen el fin de crear bienes o servicios en una economía para satisfacer necesidades de una sociedad.

El ingreso de la población mayor de 60 años a las actividades productivas es cada vez menos solicitado; sin embargo, el proyecto del INAPAM promueve la inclusión social de las personas que desean desempeñar la actividad de empacador voluntario en tiendas de autoservicio. Este proyecto busca que las personas debido a su experiencia, nivel educativo o régimen de jubilación puedan trabajar y tener un ingreso mayor al que cuentan en la actualidad. El objetivo del INAPAM es que la persona no dependa de su familia y tenga una fuente de ingresos para costear su nivel de vida. Entre las ventajas que se establecen para las empresas se encuentran las siguientes: mayor valoración del empleo, estabilidad laboral, disminución de la rotación del personal, puntualidad, ambiente laboral agradable y experiencia y desarrollo humano (INAPAM, 2019).

Este artículo analizó las características de las personas de la tercera edad que, debido a su condición económica, de salud, nivel educativo y familiar deben mantenerse o reingresar al mercado laboral para obtener ingresos que permitan sortear con mayor ligereza las vicisitudes de la vida que enfrentan a su edad. Se pretende contestar a la interrogante de: ¿Cuáles son los principales motivos por los cuales una persona considerada como jubilada o con la edad para jubilación aún se encuentra realizando actividades económicas?

\section{Metodología}

El artículo es un estudio de tipo cualitativo y cuantitativo, se enfocó en utilizar la entrevista como medio de obtención de información de 50 personas inscritas en el programa de empacadores voluntarios que tiene la oficina del INAPAM Ciudad del Carmen, Campeche, México. Estas personas se encuentran como ayudantes de caja de cobro en cuatro centros comerciales de la ciudad.

El cuestionario consistió en identificar tres aspectos fundamentales. La información considera datos demográficos (sexo, edad, nivel de estudios, dependientes económicos), elementos de trabajo (años desarrollando la actividad de empacador, razones para iniciar y mantenerse en la actividad, ingresos percibidos, clientes atendidos promedio), salud de la persona (enfermedades reportadas, estado de salud actual, problemas diarios para desarrollo de la actividad). 
El estudio es un acercamiento a seis facetas relacionadas con la calidad de vida publicadas por la OMS en 1996, los elementos analizados fueron: dolor, malestar y cansancio (esfera física) movilidad y capacidad de trabajo (grado de independencia) y la disposición de recursos financieros (esfera entorno). El estudio no abarcó las facetas correspondientes a la esfera psicológica, espiritual y de relaciones sociales debido al tiempo requerido para hacer entrevistas a profundidad y el enfoque psicológico que requería el mismo. Esta investigación parte de la idea central que los adultos mayores con una disposición de ingresos pueden resolver las demás facetas de manera parcial. La disposición de una fuente de ingresos permite al individuo poder comer, pagar servicios médicos, viajar y tener esparcimiento, le da seguridad y acceso a bienes y servicios que le permiten tener calidad de vida.

Aunque no se planteó analizar calidad de vida en todos sus factores, el análisis de cada una de las variables establecidas en el cuestionario se hizo de forma detallada. Para complementar el análisis, se realizó un modelo de regresión lineal múltiple para identificar los factores asociados al ingreso que perciben los empacadores mayores de 60 años. El modelo de regresión lineal múltiple sugerido por Pat, Martínez, Pat y Martínez (2013) y Pérez, Rivas, Martínez y Venegas (2018) se representa como:

$$
\mathrm{Y}=\beta_{0}+\beta_{1} \mathrm{X}_{1}+\beta_{2} \mathrm{X}_{2}+\mu
$$

El cual indica la relación entre una variable dependiente $(\mathrm{Y})$ y dos o más variables independientes $\left(\mathrm{X}_{1}, \mathrm{X}_{2}\right)$, además de un término error $(\mu)$. Este modelo estadístico expresa una relación lineal imperfecta entre las variables $Y$ y $X$, para ello se obtendrán estimadores $\left(\beta_{\mathrm{i}}\right)$ que permiten predecir con cierta confiabilidad el comportamiento de $Y$ (Infante y Zárate, 2011: 471).

\section{Resultados}

La incorporación de los adultos mayores en el mercado laboral está relacionada con su entorno económico y por variables de decisión propias. El proyecto permitió reconocer la importancia que tiene para una persona mayor de 60 años recibir ingresos por una actividad realizada y ser independiente de su familia; y es que a medida que pasan los años, la esperanza de vida aumenta y los sistemas de pensión y ahorro para los adultos mayores son menos eficaces para asegurar sustento a los individuos. Román, Garay y Montes de Oca (2018) afirman que la población adulta enfrenta un panorama desfavorable porque la mayoría no cuenta con ingresos fijos ni 
con una seguridad social. Damián (2016: 151) reafirma que el escenario de la pobreza existente para la población en edad de retiro tiene relación con el acceso a las pensiones, que si bien cuentan con un sistema de retiro en ocasiones los ingresos son muy bajos.

Dentro de la población entrevistada se contabilizaron 26 hombres y 24 mujeres. El grupo mostró una jornada de cuatro horas en promedio, aunque los hombres cubren jornadas de hasta ocho horas, mientras que las mujeres trabajan como máximo seis. Este aspecto se relaciona con la cantidad de dependientes económicos que tiene cada grupo de análisis, los varones apoyan a más de una persona para solventar gastos, mientras que las mujeres reportan en promedio 0.6 dependientes económicos (Tabla 2).

Tabla 2: Resumen de las principales variables analizadas en adultos mayores, 2018

\begin{tabular}{lcccccc}
\hline & \multicolumn{3}{c}{ Hombres } & & & Mujeres \\
& $\begin{array}{c}\text { Valor } \\
\text { Mínimo }\end{array}$ & Promedio & $\begin{array}{c}\text { Valor } \\
\text { Máximo }\end{array}$ & $\begin{array}{c}\text { Valor } \\
\text { Mínimo }\end{array}$ & Promedio & $\begin{array}{c}\text { Valor } \\
\text { Máximo }\end{array}$ \\
\hline Edad & 60 & 69 & 85 & 50 & 67 & 85 \\
Escolaridad & 0 & 8.5 & 12 & 0 & 6.2 & 10 \\
Horas laboradas & 4 & 5 & 8 & 4 & 4.7 & 8 \\
$\begin{array}{l}\text { Dependientes } \\
\text { Años dedicados }\end{array}$ & 0 & 1.3 & 4 & 0 & 0.6 & 2 \\
a la actividad & 1 & 4 & 10 & 1 & 3 & 5 \\
\hline
\end{tabular}

Fuente: elaboración propia.

El aporte de ingresos por parte del varón hacia la familia prevalece como una obligación social debido a la dependencia económica de la esposa u otros familiares, después de terminar su vida laboral activa, el hombre continúa buscando una fuente de ingresos. Algunos entrevistados mencionaron el desarrollo de esta función como medio de subsistencia por más de diez años, mientras que las mujeres señalaron un máximo de cinco. El trabajo deja de ser intrínseco al género masculino, las mujeres al quedar viudas o su compañero no puede desarrollar actividades económicas, deben iniciar en la búsqueda de una fuente de ingresos. Sin embargo, los varones destinan más tiempo para desarrollar la actividad. De acuerdo con Hernández (2016: 185) es la característica que legitima a los hombres como tales, el hecho que un varón pueda proveer y mantener a una familia implica un reconocimiento de la sociedad y de la propia familia.

Los empacadores de la tercera edad se sienten útiles y agradecidos con las tiendas que les permiten desarrollar la actividad; sin embargo, la inexis- 
tencia de un contrato con los supermercados para obtener un ingreso también limita su calidad de vida al generar un estrés por depender de la cantidad y generosidad de los clientes atendidos; al considerar un promedio de cuatro horas de trabajo, los ingresos oscilan entre 100 y 140 pesos; el ingreso puede aumentar conforme se amplía el tiempo llegando a alcanzar 320 pesos en una jornada completa de trabajo, es decir, manteniéndose las ocho horas como empacador en una estación de cobro (Tabla 3); en palabras de un entrevistado de 66 años (núm. 33):

... mira hay días que nos va muy bien, nos llevamos unos doscientos pesos, pero hay días que solo sacamos unos treinta o cuarenta pesos porque estamos dos en una caja. Además, que solo nos dejan estar cuatro horas porque están nuestros compañeros que también les toca.

Tabla 3: Ingresos promedio acordes a las jornadas de trabajo, 2018

\begin{tabular}{lrrrr}
\hline Variables & \multicolumn{4}{c}{ Valores } \\
\hline Horas como empacador & 4 & 5 & 6 & 8 \\
Ingresos promedio & 138 & 169 & 226 & 320 \\
Cantidad de clientes atendidos promedio & 37 & 40 & 56 & 98 \\
\hline
\end{tabular}

Fuente: elaboración propia.

La generación de ingresos por parte de los empacadores depende de la cantidad de clientes atendidos, por lo cual, una ampliación en los horarios podría permitirles tener un mayor ingreso; sin embargo, las personas de este grupo poblacional tienen diferentes padecimientos que les impiden permanecer más de seis horas de pie, el mantenerse jornadas largas implica que demeritan y agravan su situación de salud. La solución de trabajar parece no ser la mejor forma de mejorar su calidad de vida, sino por el contrario la disminuye, el aumento de dolores en las piernas y molestias de las manos por cuestiones de artritis se hace presente cuando más tiempo se dedican a empacar productos. De esta manera la solución a la falta de ingresos parece convertirse en una causa de tener que seguir trabajando para poder cubrir otros gastos que implica un deterioro en el estado de salud y económico de la persona.

El modelo lineal relaciona ingreso percibido, horas laboradas y número de personas atendidas. Por una hora laborada se obtienen 31 pesos y aumenta en promedio 1.06 pesos por cada cliente que ayuda a empacar sus compras (Tabla 4). En general, los empacadores voluntarios obtienen mejores ingresos durante los días de mayor actividad comercial (martes, 
sábado y días de quincena); sin embargo, implica más cansancio y estrés para las personas que padecen problemas de salud. Los entrevistados mencionan que, durante los días más concurridos a los supermercados, los clientes muestran mayor disposición a otorgar más dinero al recibir el servicio. En promedio, los ingresos durante estos días incrementan cerca de 15 por ciento.

Tabla 4: Coeficientes del modelo lineal de ingresos, 2018

\begin{tabular}{|c|c|c|c|c|}
\hline \multirow[t]{2}{*}{ Modelo } & \multicolumn{2}{|c|}{ Coeficientes } & \multirow[b]{2}{*}{$\mathrm{t}$} & \multirow[b]{2}{*}{ Probabilidad } \\
\hline & B & Error típico & & \\
\hline Intercepto $(B o)$ & -29.5439 & 41.4820 & -0.7122 & 0.4798 \\
\hline Horas laboradas $(B 1)$ & 31.6492 & 10.4852 & 1.8687 & 0.0040 \\
\hline Personas atendidas (B2) & 1.0633 & 0.5689 & 3.0262 & 0.0678 \\
\hline
\end{tabular}

Se considera un $\alpha=0.05$ y coeficiente de determinación $\mathrm{R}^{2}$ de 0.40

Fuente: elaboración propia.

De acuerdo con su nivel de estudio (Tabla 5), la mitad de las personas - sin importar el sexo- tienen estudios de primaria y son el grupo más numeroso que se encuentra ejecutando esta actividad (48 por ciento). Las personas con poca formación educativa que trabajaron durante su vida económica en actividades informales o con pocas prestaciones, no tienen una cuota determinada para su jubilación o los ingresos son bajos, por lo que se ven obligados a seguir desarrollando actividades ya sea de manera formal o informal para conseguir sueldos o gratificaciones. El caso de los empacadores no se considera un salario; sin embargo, las personas de la tercera edad ven la actividad como un empleo formal que exige asistencia, puntualidad, dedicación y destreza para desarrollarlo.

Tabla 5: Distribución de la muestra de acuerdo al nivel de estudio y sexo, 2018

\begin{tabular}{lrrrr}
\hline Nivel de estudio & Hombre & Mujer & Total & Porcentaje \\
\hline Sin estudios & 1 & 2 & 3 & 6 \\
Primaria incompleta & 1 & 2 & 3 & 6 \\
Primaria & 11 & 13 & 24 & 48 \\
Secundaria & 9 & 4 & 13 & 26 \\
Preparatoria & 4 & 3 & 7 & 14 \\
Total & 26 & 24 & 50 & 100 \\
\hline
\end{tabular}

Fuente: elaboración propia. 
La desigualdad en el acceso a la educación para este sector es marcada. En la muestra, los hombres tienen mayor nivel de estudio, mientras que las mujeres demuestran la poca posibilidad de asistencia a la escuela durante su niñez. El INAPAM sugirió el proyecto de empacador voluntario como una forma de obtener ingreso para los adultos mayores que por su nivel de educación y sus capacidades no reciben una jubilación digna. Esa forma ha sido copiada por otras tiendas que han aceptado el ingreso de personas de la tercera edad como empacadores, pero sin ninguna responsabilidad para con ellos, solo brindarles la posibilidad de obtener propinas por parte de los clientes (Tabla 6).

Tabla 6: Nivel de escolaridad y jornadas cubiertas por los empacadores voluntarios, 2018

\begin{tabular}{llcllr}
\hline & \multicolumn{5}{c}{ Horas cumplidas como empacador } \\
Escolaridad & 4 & 5 & 6 & 8 & Total \\
\hline Sin estudios & 0 & 3 & 0 & 0 & 3 \\
Primaria incompleta & 2 & 1 & 0 & 0 & 3 \\
Primaria & 6 & 11 & 7 & 0 & 24 \\
Secundaria & 5 & 7 & 0 & 1 & 13 \\
Preparatoria & 2 & 4 & 1 & 0 & 7 \\
Total & & & & & 50 \\
\hline
\end{tabular}

Fuente: elaboración propia.

Las políticas sociales de México intentan apoyar a toda la población mayor de 60 años por medio de subsidios económicos; sin embargo, la realidad indica que los montos recibidos no son suficientes para cubrir todos los gastos que tienen y surge la necesidad de acudir a los centros de apoyo al adulto mayor para inscribirse en el programa de empacador voluntario, única actividad que les permite percibir una propina.

Es decir, a mayor edad, sin importar el nivel de educación, existen otros elementos que obligan a la persona estar desarrollando una actividad para hacerse llegar de ingresos. De acuerdo a un estudio realizado por Román-Sánchez et al. (2019:102) encontró que nueve de cada diez adultos mayores que están en edad de retirarse no lo hacen ya sea por obligación o por necesidad. Además, Canto (2019: 1181) señala que el salario mínimo que recibe un trabajador en México no alcanza para comprar la canasta básica individual. Estos elementos obligan a las personas a continuar laborando a pesar de tener la edad para retirarse del mercado laboral. De acuerdo con los resultados obtenidos, las personas entre 63 y 72 años son 
las más activas como empacadores en las tiendas de autoservicios y en su mayoría cubren entre cuatro y seis horas (Figura 1).

Entre los motivos de trabajo, 90 por ciento de los entrevistados indicaron la actividad como una necesidad, la mayoría aseguró tener ingresos bajos que no solventan sus gastos de alimentación, vivienda y la compra de medicamentos. En comentarios de los entrevistados se resaltaba el efecto de la inflación en los precios de los alimentos y principalmente de las medicinas; este aumento de precios obliga a la población a continuar buscando un medio para hacerse llegar de ingresos. Siendo el trabajo como empacador uno de los únicos que no solicita muchos requisitos.

Dentro de la información que se dispone en el Diario Oficial de la Federación del 16 de diciembre de 2020, en el artículo 170 indica que una persona con más de 65 años al momento de retirarse con mil semanas de cotización y considerando menos de dos unidades de medida y actualización (UMA) puede recibir 2,809 pesos mensualmente (DOF, 2020), esta cantidad no podría cubrir las necesidades básicas de una pareja de adultos mayores si dependieran únicamente de ese ingreso para vivir. Este nivel de pensión corresponde a personas con puestos de trabajos de bajo nivel como ayudantes generales y empleados con niveles de educación correspondientes a la primaria. En el caso del estudio, 60 por ciento de los entrevistados habían estudiado hasta la primaria, 26 por ciento tenían secundaria y solo 14 por ciento habían cursado la preparatoria. Considerando los niveles de pensión indicados para personas con niveles básicos de educación, los ingresos para la población entrevistada no superaban los 4,800 pesos mensuales. La necesidad de cubrir el faltante de sus ingresos mediante un trabajo parcial como es el caso de los empacadores, se presenta como una solución a la falta de ingresos por parte de los adultos mayores.

Una de las observaciones que hacían los entrevistados es referente a las limitaciones del mercado de trabajo, en palabras de una entrevistada (núm. 9) de 67 años al responder a la pregunta: ¿Por qué trabaja como empacadora?: "Es lo único que hay para nosotros, ya no nos dan trabajo porque creen que los viejos no servimos para trabajar".

Esta respuesta fue muy común en todos los entrevistados que indicaron trabajar por necesidad y es que, aunque tienen el interés en desarrollar otras actividades, el mismo mercado de trabajo limita su posibilidad de ingresar a otras áreas para poder desarrollarse y contribuir a un proceso productivo.

Damián (2016) consideró que para 2014, del total de la población nacional mayor de 60 años, 37 por ciento se declaró ocupado y de estos, 58.8 por ciento no recibía una pensión. 
Vinculación laboral en personas de la tercera edad: el caso de los empacadores ... / A. PÉREZ FERNÁNDEZ y J.A. VENEGAS VENEGAS

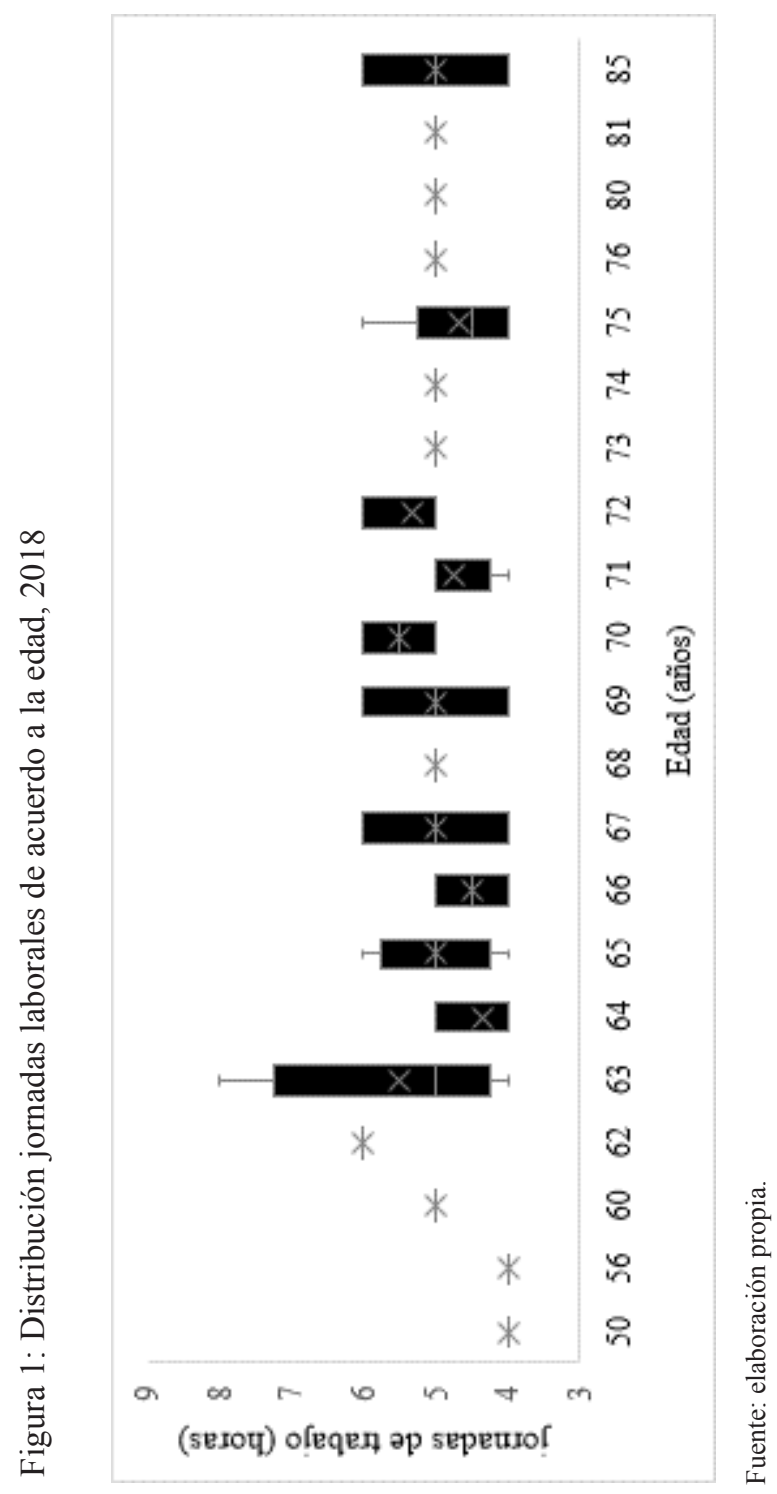


Este último apartado cobra importancia debido al incremento de problemas de salud que reporta este sector de la población y sin ingresos suficientes no pueden hacer frente a los gastos que implican el cuidado de la salud.

Solo diez por ciento acude a trabajar porque consideran el desarrollo de una actividad fuera del hogar como un medio para alcanzar mayor plenitud. En general, este grupo de personas cubren turnos cortos (4 - 5 horas) y son personas que cuentan con pensión, apoyo gubernamental o apoyo de familiares. La posibilidad de salir de la monotonía hogareña, tener nuevas amistades y obtener un ingreso extra por mantenerse ocupado son algunas de las razones para este grupo de trabajadores. Las personas de este subgrupo son mayormente individuos que han desarrollado actividades durante toda su vida y la monotonía no la consideran como parte de su desarrollo como individuo después de separarse de sus actividades productivas, además que la mayoría de las personas que desean salir a trabajar por deseo propios, es por el hecho de vivir solos en casa, ya sea porque los hijos se han ido a otras ciudades o algunos de ellos han perdido a la pareja que era parte de la compañía.

El estado de salud y su relación con la productividad de los trabajadores es importante en el sistema económico; sin embargo, los adultos mayores son más propensos a padecer enfermedades propias de la edad. Las personas entrevistadas indicaron tener problemas asociados a la diabetes, al reumatismo y la presión arterial alta (Figura 2). Estas enfermedades traen consigo cambios en el rendimiento del trabajador por los problemas indirectos que provocan como ausentismo y falta de energía para el desarrollo de las actividades del proceso. Dentro del horario de trabajo, las personas presentan dolores musculares, dolores de articulaciones, inflamación de pies y cansancio general asociado a sus enfermedades crónicas o al simple desarrollo de la actividad. La jornada de cuatro horas propuesta por el INAPAM es propia para una persona que no puede mantenerse un largo periodo de pie; sin embargo, existen personas que prefieren laborar las ocho horas para aumentar las propinas otorgadas por los clientes de las empresas. La necesidad de abarcar periodos largos a pesar de presentar algún problema de salud, demuestra la falta de un programa de seguridad gubernamental para las personas que no disponen de un ingreso suficiente para mantener un nivel de vida aceptable. 
Figura 2: Problemas de salud reportadas por adultos mayores, 2018

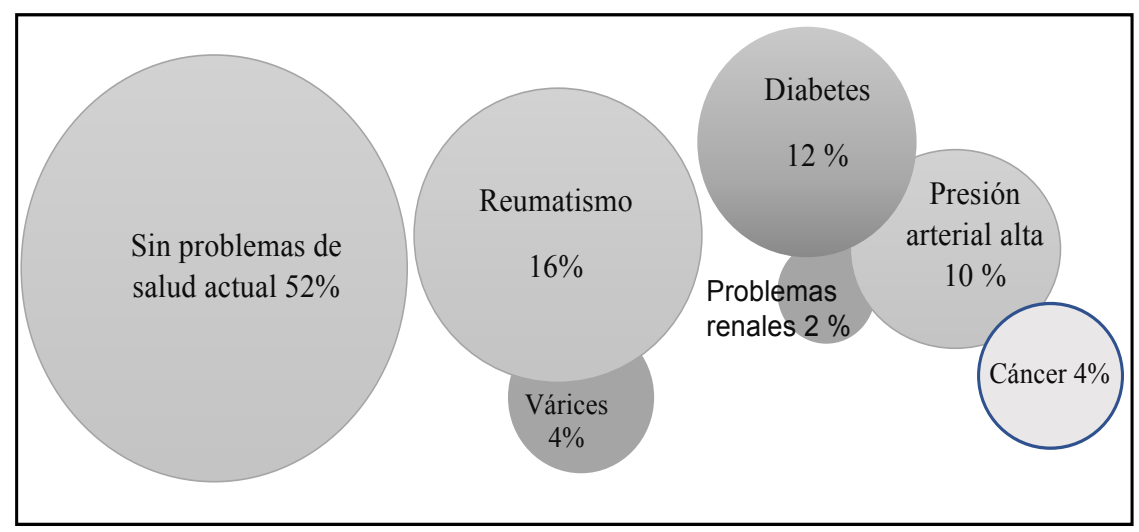

Fuente: elaboración propia.

En los elementos analizados, los adultos mayores reflejan la necesidad de desarrollar una actividad económica para obtener un ingreso o mantenerse activos físicamente; y es que, en estudios se muestra que las personas aceptan funciones dentro de una empresa $u$ hogar debido a la satisfacción que sienten de poder ayudar. De los Santos y Carmona (2018) evidenciaron que la ocupación y las actividades sociales son factores estadísticamente asociados a la aparición de depresión en personas mayores. La salud de las personas implica no solo dolor físico, sino procurar que se sientan útiles y sin depresión en su vida.

La obtención de ingresos para adultos mayores depende del trabajo que desarrollaban, el nivel de estudios, el área geográfica en la que viven y su dependencia de hijos o familiares. Sin embargo, cuando el ingreso es menor a los gastos a cubrir, es necesario que las personas desarrollen alguna actividad para financiar los egresos faltantes. El análisis permitió identificar seis facetas que forman parte de la calidad de vida de 50 adultos mayores de Ciudad del Carmen, Campeche; 98 por ciento considera la movilidad como un elemento de alta importancia, la población que no depende de aparatos o personal médico para moverse, se considera útil, con habilidades y capacidades para desarrollar un trabajo. Los entrevistados reconocen tener la capacidad de trabajo ya sea en la casa donde habitan o realizando actividades retributivas fuera del hogar, como es el caso de los empacadores de las tiendas de autoservicio, que a su vez les genera una independencia financiera. 
La libertad de movimiento que indican tener los empacadores es limitada, debido a los problemas de salud como la artritis, cansancio al estar mucho tiempo de pie o limitaciones para poder cargar elementos pesados; sin embargo, son condiciones que les permite realizar actividades de manera cotidiana y en el caso de las personas que no disponen de un ingreso vitalicio, pueden destinar el tiempo y la energía para obtener propinas al ser empacadores en los supermercados.

Yánez, Maldonado y Del Risco (2016) indican que esta población tiene altos índices de informalidad y bajas condiciones laborales. Además, indican que el ser hombre, jefe de hogar, poseer cualquier nivel educativo diferente al nivel universitario aumenta la probabilidad de participar en actividades laborales. Cuando no es posible su inserción al sistema económico, iniciar un negocio propio puede ser una buena forma de hacer frente a la nueva etapa de la vida. Oelckers (2015) considera el emprendimiento como una alternativa viable para mejorar el ingreso y aminorar la desintegración del adulto mayor de la vida laboral de una sociedad.

La poca calidad de vida que muestran los adultos mayores por falta de recursos financieros para solventar sus gastos ha implicado que el Gobierno de México reconozca la necesidad de revisar las políticas públicas enfocadas en este grupo poblacional. En un comunicado del 21 de marzo de 2021, el presidente de México, Andrés Manuel López Obrador, anunció lo siguiente:

a. La pensión universal para adultos mayores se entregará a partir de los 65 años, como se hace cuando se trata de población indígena.

b. La actual pensión se incrementará gradualmente hasta llegar al doble al inicio del 2024.

c. Este plan iniciará desde julio del presente año con un incremento del 15 por ciento. En enero de 2022, 2023 y 2024 el aumento será de 20 por ciento anual más inflación hasta llegar a seis mil pesos bimestrales (Gobierno de México, 2021).

El objetivo de la modificación de esta política es permitir a más de diez millones de personas adultas mayores de tener acceso a un ingreso que les permita mejorar su calidad de vida.

\section{Conclusiones}

Las personas de la tercera edad ingresan al mercado laboral debido a que, aunque cuentan con apoyo gubernamental o familiar, el ingreso percibido 
no les alcanza para solventar los gastos realizados, en general, el programa de empacadores voluntarios es una oportunidad para las personas que requieren seguir trabajando para mantener un ingreso estable. En general, se pudo apreciar que las personas no sienten que sean valoradas por el trabajo que realizan. Aponte (2015: 154) considera que las actividades que rodean al adulto mayor en la mayoría de los casos son desmoralizadoras, una persona debe ser valorada desde la presencia de condiciones materiales y espirituales.

La sociedad requiere de los programas gubernamentales asistencialistas para brindar un ingreso permanente a los grupos de más de 60 años que por su capacidad productiva son poco seleccionados para ingresar a los sistemas productivos. Flores y Salas (2018) encuentran que el grupo etario se desarrolla en condiciones cada vez más precarias, lo cual demuestra la parcial respuesta proveniente de las políticas públicas asociadas a este grupo.

El cumplimiento de jornadas largas para obtener ingresos que permitan cubrir gastos inmediatos conlleva una baja calidad de vida en los adultos mayores, debido a que, las enfermedades como reumatismo, varices, diabetes y cansancio corporal propios de la edad hacen el periodo de trabajo más tedioso y disminuyen su capacidad de trabajo. Las personas intercambian bienestar físico por bienestar económico. La deshumanización del trabajo se hace presente en todas las etapas del trabajador.

\section{REFERENCIAS BIBLIOGRÁFICAS}

Alessandrini, G. R. y Sánchez R. K., 2007, Tercera edad: consideraciones éticas. Bioetica. Consultado el 22/03/2019 disponible en http://www.cbioetica.org/revista/73/730409.pdf

Aponte, D. V. C., 2015, "Calidad de vida en la tercera edad", en Ajayu 13(2):152-182. Disponible en http://www.scielo.org.bo/scielo.php?pi$\mathrm{d}=$ S2077-21612015000200003\&script=sci_arttext

Arcia, M de O., 2004, "Participación en la fuerza laboral de los adultos mayores en Latinoamérica y el Caribe", en Carta Económica Regional 27(115): 27-35. Consultado el 28/01/2018, disponible en http://revistascientificas.udg.mx/index. php/CER/article/view/5730/5250

Bruno, F. y Acevedo, A. J., 2016, "Vejez y sociedad en México: Las visiones construidas desde las Ciencias Sociales", en Forum sociológico (29) consultado el 8/01/2019, disponible en http://journals.openedition.org/sociologico/1453; DOI : $10.4000 /$ sociologico. 1453

Canto, S. R. A., 2019, “¿Distribuir o redistribuir? Pobreza, desigualdad y política pública en México", en Economía, Sociedad y Territorio. 18 (59), 1181-1211. Doi: http://dx.doi.org/10.22136/est20191259 
CONAPO, 2019, Indicadores de en la población de 60 años y más estimados con información de las proyecciones de la población, 1970-2050. Consejo Nacional de Población (CONAPO), consultado el 08/01/2019, disponible en https://datos. gob.mx/busca/dataset/envejecimiento-demografico

Damián, A., 2016, "Seguridad social, pensiones y pobreza de los adultos mayores en México", en Acta Sociológica, 70(1): 151-172. Doi: https://doi.org/10.1016/j. acso.2017.01.007

De los Santos, P. V. y Carmona V. S. E., 2018, "Prevalencia de depresión en hombres y mujeres mayores en México y factores de riesgo", en Población y Salud en Mesoamérica, 15(2): 1-23, Doi: https://doi.org/10.15517/psm.v15i2.29255

Diario Oficial de la Federación, 2020, Decreto por el que se reforman, adicionan $y$ derogan diversas disposiciones de la Ley del Seguro Social y de la Ley del Sistemas de Ahorro para el retiro. 14 de diciembre de 2020, https://www.dof.gob.mx/ nota_detalle.php?codigo $=5607729 \&$ fecha $=16 / 12 / 2020$

Durán, S., Prieto, R. y García J., 2017, “Influencia de la calidad de vida en el rendimiento del estudiante universitario", en Hernández G. de Velazco, J. (coordinadora). Calidad de vida, inclusión social y bienestar humano. Colección sin Fronteras (UNERMB. Consultado el 20/01/2019, disponible en http://150.185.9.18/fondo editorial/images/PDF/CSF $/ 00 \% 20$ Calidad $\% 20 \mathrm{de} \% 20$ Vida $\% 20$ Inclusin $\% 20 \mathrm{y} \% 2 \overline{0}$ Bienestar\%20Social\%20-\%20Hernndez\%20Barboza\%20Muoz\%20compiladores\%201.pdf\#page $=107$

Esquivel, H. M. T. y Sánchez-Mejorada, 1995, “Condiciones de vida y política social para la población de la tercera edad”, en Sociológica 10(29). Consultado el 26/11/2018, disponible en http://www.sociologicamexico.azc.uam.mx/index.php/ Sociologica/article/view/661/634

Filgueira, F. y Espíndola E., 2015, Hacia un sistema de transferencias monetarias para la infancia y los adultos mayores. Una estimación de impactos y posibilidades fiscales en América Latina, en Serie Políticas sociales. CEPAL, Naciones Unidas. Ministerio de Asuntos Exteriores de Noruega. Consultado el 7/01/2019, disponible en http://repositorio.minedu.gob.pe/bitstream/handle/123456789/3527/ Hacia $\% 20$ un $\% 20$ sistema $\% 20$ de $\% 20$ transferencias $\% 20$ monetarias $\% 20$ para $\% 20$ la\%20infancia\%20y\%20los\%20adultos\%20mayores\%20una\%20estimaci\%C3\%B3n\%20de \%20impactos\%20y\%20posibilidades\%20fiscales $\% 20$ en $\% 20$ Am\%C3\%A9rica\%20Latina.pdf?sequence=1\&isAllowed $=\mathrm{y}$

Flores, P. L., y Salas, D. I.A., 2018, “Calidad del empleo en grupos socialmente vulnerables en México. El caso de los adultos mayores", en Economía, sociedad y territorio. 18(56): 1-33. Doi: http://dx.doi.org/10.22136/est20181066

Gobierno de México, 2021, Comunicado: En Guelatao, presidente anuncia ampliación y aumento a pensión universal de adultos mayores. 21 de marzo de 2021, disponible en https:/www.gob.mx/presidencia/prensa/en-guelatao-presidente-anuncia-ampliacion-y-aumento-a-pension-universal-de-adultos-mayores?idiom $=$ es 
Hernández, H. O. M., 2016, “Trabajo y construcción de masculinidades en el norte de México", en Frontera Norte, 28(55): 183-189. Disponible en https://fronteranorte.colef.mx/index.php/fronteranorte/article/view/37/53

INAPAM, 2019, Vinculación productiva para personas adultas mayores. Consultado el 9/01/2019, disponible en https://www.gob.mx/inapam/acciones-y-programas/vinculacion-productiva-para-personas-adultas-mayores

Infante, G. S. y Zárate de L. G. P., 2011, Métodos estadísticos: un enfoque interdisciplinario. Ed. Trillas 1990 (reimp 2011). México D. F. 643 p.

Levaggi, V., 2004, ¿Qué es el trabajo decente? Organización Internacional del Trabajo consultado el 3/07/2019, disponible en https://www.ilo.org/americas/sala-de-prensa/WCMS_LIM_653_SP/lang--es/index.htm

Llanes, T.H.M., López S.Y., Vázquez, A.J.L. y Hernández, P. R., 2015, “Factores psicosociales que inciden en la depresión del adulto mayor", en Revista de ciencias médicas de la Habana, 21(1): 65-74.

Limón, M. R. y Ortega M. del C., 2011, "Envejecimiento activo y mejora de la calidad de vida en adultos mayores", en Revista de psicología y Educación, vol. 6: 225-238. Consultado el 28/01/2019, disponible en http://www.rpye.es/pdf/68.pdf

Maldonado, B.J.J. Corrales V. J. M., Ruíz F. M. I. y González B. S., 2016, "Nuestros mayores activos: inteligentes y emprendedores", en International Journal of Developmental and Educational Psychology, 2: 85-92.

Navarro, O. A., 2017, "Vulnerabilidad, trabajo y salud en mujeres de la tercera edad en Ameca, Jalisco", en Región y Sociedad, 29(68): 5-36. Doi: http://dx.doi. org/10.22198/rys.2017.68.a223

Oelckers, F., 2015, "Emprendimiento en la tercera edad: una revisión de la situación actual", en Journal of Technology management\& innovation. 10(3):143-153: Doi: http://dx.doi.org/10.4067/S0718-27242015000300015

Organización Mundial de Salud, 1996, La gente y la salud. Comunicado del Foro Mundial de la Salud. vol. 17. Consultado el 10/01/2019, disponible en http://apps. who.int/iris/bitstream/handle/10665/55264/WHF_1996_17_n4_p385-387_spa. pdf? sequence $=1 \&$ isAllowed $=\mathrm{y}$

OMS, 2018, Envejecimiento y salud. Organización Mundial de Salud (OMS), Consultado el 21/03/2019, disponible en https://www.who.int/es/news-room/factsheets/detail/envejecimiento-y-salud

Pat, F. L.A., Martínez, M. A. H., Pat, F. J.M. y Martínez L. D., 2013, Introducción a los modelos de regresión. Plaza y Valdés- UNACAR, Campeche, México.

Pérez, F. A., Rivas, M. M. I., Martínez L. D. y Venegas, V. J. A., 2018, "Reestructuración de Pemex y su efecto en los salarios y el mercado de trabajo en Ciudad del Carmen, Campeche", en Análisis Económico, 82(33): 111-124. Disponible en http://analisiseconomico.azc.uam.mx/index.php/rae/article/view/36/35 
Rendón, T., 2004, "El mercado laboral y la división intrafamiliar del trabajo", en M. Ariza, y O. De Oliveira (Eds.) Imágenes de la familia en el cambio de siglo. México: Universidad Nacional Autónoma de México.

Román, M. A., Garay V. S., y Montes de Oca Z. V., 2018, “Cambios y permanencias en la población beneficiaria del Programa de Apoyo Directo al Adulto Mayor en Nuevo León 2004-2014", en Región y sociedad, 30(72): 1-32. DOI: https:// dx.doi.org/10.22198/rys.2018.72.a787

Román-Sánchez, Y. G., Montoya- Arce, B. J., Gaxiola-Robles, L. S. C. y Lozano-Keymolen D., 2019, "Los adultos mayores y su retiro del mercado laboral en México", en Sociedad y Economía, 37, 87-113. Doi: https://doi.org/10.25100/ sye.v0i37.7823

Romano, C. G., 2013, “Adultos mayores: nuevos paradigmas del derecho y la seguridad social”, en Letras jurídicas, 17: 1-27.

Rubio, O. D. Y., Rivera M. L., Borges O. L de la C. y González C. F. V., 2015, “Calidad de vida en el adulto mayor", en VARONA 61: 1-7. Consultado el 9/01/2019, disponible en https://www.redalyc.org/html/3606/360643422019

Villasmil, M. M., Romero, F. J., Chivetta, L. E. y Palacios A., 2017, “Efectividad de las contribuciones parafiscales como estrategia de la inversión en la seguridad social", en Espacios 38(43): 1-14, consultado el 10/01/2019, disponible en http://bonga.unisimon.edu.co/bitstream/handle/123456789/1741/Efectividad\%20 de $\% 201$ as $\% 20$ Contribuciones.pdf? sequence $=1 \&$ is Allowed $=y$

Yánez, C. M. A., Maldonado P. C. D. y Del Risco, S. K. P., 2016, "Participación laboral de la población de 60 años de edad o más en Colombia", en Revista de Economía del Caribe, 17: 39-63 consultado en http://www.scielo.org.co/pdf/eco$\mathrm{ca} / \mathrm{n} 17 / \mathrm{n} 17 \mathrm{a} 02 . \mathrm{pdf}$

\section{RESUMEN CURRICULAR DE LOS AUTORES}

\section{Alberto Pérez Fernández}

Maestro en Ciencias en Economía Agrícola y de los Recursos Naturales y Doctor en Ciencias en Economía Agrícola por la Universidad Autónoma Chapingo. Desde 2015 se desempeña como profesor de tiempo completo en la Facultad de Ciencias Económicas- Administrativas de la Universidad Autónoma del Carmen. Líder de la academia de Economía, miembro del Cuerpo Académico Economía y Administración de Empresas. Es miembro del Sistema Nacional de Investigadores (nivel 1). Las líneas de investigación son economía sectorial emergente, mercado de trabajo y desarrollo económico. Entre sus últimas publicaciones se encuentra: Reestructura- 
Vinculación laboral en personas de la tercera eddad: el cass de los empaceadores ... / A. PÉREZ FERNÁNDEZ y J.A. VENEGAS VENEGAS

ción de Pemex y su efecto en el mercado de trabajo en Ciudad del Carmen, Campeche.

Dirección electrónica: apfernandez@pampano.unacar.mx

Registro ORCID: https://orcid.org/0000-0002-9397-4167

José Apolonio Venegas Venegas

Maestría en Economía por el Colegio de Postgraduados. Doctor en Economía Agrícola por la Universidad Autónoma Chapingo. Es Catedrático CONACYT-UNACH desde el 1 de noviembre de 2015 en la Facultad de Ciencias Agronómicas de la Universidad Autónoma de Chiapas, donde participa en los programas de la Maestría en Producción Agropecuaria Tropical (MCPAT) y Doctorado en Ciencias Agropecuarias y Sustentabilidad (DOCAS). Miembro colaborador del Cuerpo Académico de Agroforestería Pecuaria (consolidado). Pertenece al Sistema Nacional de Investigadores Nivel I y al Sistema Estatal de Investigadores. Las líneas de investigación que desarrolla son energía renovable y economía agrícola. Desarrollo económico y mercado emergentes.

Dirección electrónica: polo_tex1@hotmail.com

Registro ORCID: https://orcid.org/0000-0002-3855-8355

Artículo recibido el 7 de agosto de 2019 y aprobado el 5 de mayo de 2020. 